chemotherapeutic agents. Determine immunohistochemical NFKB expression in tissue microarrays of spontaneous canine neoplasms and correlate with outcome-linked data. Characterize the in vivo sensitivity of canine hematopoietic cell lines to PTL using a murine xenograft model. METHODS/STUDY POPULATION: Growth inhibition assays were performed using a panel of canine mast cell, histiocytic sarcoma, lymphoma, and leukemia cell lines, with PTL alone or in combination with redox-perturbing standard-of-care therapeutics. Cell death was assessed using flow cytometry. Immunofluorescence and immunoblotting were used to assess NFkB localization and phosphorylation of NFKB p65 (transcriptional activation), respectively. Intracellular glutathione with and without PTL and combination chemotherapeutics will be assessed spectrophotometrically. Archived spontaneous canine tumors will be evaluated immunohistochemically (IHC) for increased NFKB pathway activation relative to normal control tissues. Nude mice will receive intravenous, intraperitoneal, or subcutaneous injections of canine HS cells and will be treated with PTL or with PTL in combination with standard-of-care chemotherapeutics. RESULTS/ANTICIPATED RESULTS: Results: All immortalized canine cell lines evaluated are sensitive to PTL therapy and undergo dose-dependent apoptosis following exposure to drug. PTL exposure leads to inhibition of $\mathrm{NF \kappa B}$, as evidenced by immunofluorescent nuclear exclusion and decreased p65 phosphorylation. Some chemotherapeutics appear to synergize with PTL in vitro. Anticipated results: We expect to find increased IHC NFKB pathway activation in malignantly transformed tissues relative to controls. We expect standard-of-care therapeutics to synergize with PTL in vivo based on preliminary in vitro data. DISCUSSION/SIGNIFICANCE OF IMPACT: These studies will determine whether PTL therapy may be beneficial in dogs with a variety of hematopoietic neoplasms, either alone or in combination with other therapeutics that are currently in clinical use. Dogs with mast cell or histiocytic neoplasia are an excellent model for rare and deadly human diseases, which may also benefit from PTL therapy.

\section{Management of Acute Rejection in Penile Allotransplantation}

Michael Jonczyk ${ }^{1}$, Ilse M. Schol ${ }^{2}$, Philipp Tratnig-Frankl², Alexandre G. Lellouch², Dicken S.C. Ko ${ }^{3}$ and Curtis L. Cetrulo Jr. ${ }^{2}$ ${ }^{1}$ Tufts University; ${ }^{2}$ Massachusetts General Hospital and

${ }^{3}$ St.Elizabeth's Medical Center

OBJECTIVES/SPECIFIC AIMS: Objective: To summarize the diagnosis and management of two acute rejection (AR) episodes in the first penis transplant patient in the U.S. Background: Vascularized composite allotransplantation (VCA) has been utilized for stateof-the-art reconstruction of devastating craniofacial defects, limb loss, and recently, severe genitourinary defects. To date, more than 200 VCA's have been performed, of which four successful penis transplants have been achieved worldwide (Two in the U.S.). However, despite the technical success of VCAs in general, acute rejection episodes remain a significant postoperative management problem, with $80-85 \%$ experiencing at least one episode in the firstyear post-transplantation. The incorporation of skin in VCAs, which is highly immunogenic, allows early visible recognition of rejection but requires prompt management to prevent allograft failure as well as the progression of chronic rejection, which has been associated with the frequency of acute rejection episodes in preclinical models. We present the first report of acute rejection in a penile allograft. AR episodes in VCA typically manifest with erythema of the allograft skin and/or maculopapular lesions that can be either patchy, focal or diffuse. Histopathologic assessment is essential for diagnosis and management. The Banff classification of histopathologic criteria for degree of $\mathrm{AR}$ is most commonly utilized to direct clinical management. METHODS/STUDY POPULATION: We reviewed the clinical course of the first American patient who underwent penis transplantation at Massachusetts General Hospital in 2016. Postoperatively, routine clinical and chemical assessment (immunosuppression levels, routine blood work) was performed, with increased frequency during AR episodes. Skin punch biopsies were obtained during (suspected) AR episodes, analyzed and graded according to the Banff 2007 classification of rejection of skincontaining composite allografts. Histopathologic tissue assessment included CD3, C4d, CD4/8, CD20 FOXP3 and cellular infiltration (hyper keratinization, lymphocytic infiltrate, dermal erosion, macrophage, eosinophilia, T-cell infiltration) and epidermal or perivascular fibrosis. RESULTS/ANTICIPATED RESULTS: The patient is a 65-year-old male with history of penile carcinoma requiring subtotal penectomy in 2012 . He is currently 30 -months post penile transplantation (as of 11/15/2018). First Rejection Episode: At 28 days posttransplantation, the patient noted induration, swelling and erythema of the allograft, which was diagnosed as AR clinically (Image 1A). Biopsy showed a Banff Grade III AR, with focal keratinocyte apoptosis with lymphocytic infiltration in epidermis and arteriolar endothelialitis with perivascular inflammation. Initially this episode was treated for 2 days with 2 pulse doses of methylprednisolone $(500 \mathrm{mg} / \mathrm{d} \mathrm{IV})$ with clinical improvement. However, recurrent allograft erythema was observed on postoperative day 32 and an acute rejection grade III according the Banff classification was confirmed by a second biopsy that demonstrated epidermal perivascular lymphocytic infiltrates, spongiosis and dyskeratosis, deep dermis focal lymphocytic infiltrates and focal infiltrates in arterioles as well as endothelialitis in venules. Donor specific antibodies and C4d were negative. $\mathrm{CD} 3+\mathrm{T}$ cells were present in the epidermis and perivascular space. This was treated with anti-thymocyte globulin (thymoglobulin) course for 4 days $(1.5 \mathrm{mg} / \mathrm{kg} /$ day IV) and 3 more pulse doses of methylprednisolone $(500 \mathrm{mg} / \mathrm{d}$ IV.), followed by a prednisone $(250 \mathrm{mg} / \mathrm{d})$ taper to baseline. This resulted in complete resolution of AR. Second Rejection Episode: At 10.8 months post VCA the patient presented with penile erythema and scrotal swelling suggestive of AR and received three doses of methylprednisolone (day 1: $500 \mathrm{mg} / \mathrm{d}$ IV, day 2: $1000 \mathrm{mg} / \mathrm{d}$ IV and day 3: $500 \mathrm{mg} / \mathrm{d}$ IV respectively) followed by increased baseline prednisone (10 mg PO daily; increased dose compared to previous AR episode). A skin biopsy confirmed Banff Grade III AR. Compared to the previous biopsy, this biopsy demonstrated an increased density of lymphocytic inflammation of the dermis with endarteritis. Prominent involvement of epidermis and adnexal structures corresponding to acute T-cell mediated rejection was also observed (Figure 1). Donor specific antibodies and C4d were again negative. Three doses of ATG $(1.5 \mathrm{mg} / \mathrm{kg} /$ day IV) were administered. In addition, tacrolimus was increased and local tacrolimus ( $1 \%$ ointment) treatment was begun. Clinical signs of rejection improved and repeat biopsy showed dramatic histopathological improvement. Current maintenance immunosuppressive regimen consists of tacrolimus, sirolimus, prednisone, mycophenolic mofetil acid (MMF), rapamycin, and tacrolimus ointment, with no new clinical or histopathological signs of rejection (Image 1B). DISCUSSION/SIGNIFICANCE OF IMPACT: We report the first described case of acute $\mathrm{T}$-cell mediated rejection in penile transplantation. These rejection episodes demonstrated that, even on stringent immunosuppressive regimens, severe acute rejection episodes in VCA may still occur. Edema and acute induration 
preceded the development of erythema in our cases, representing a harbinger for the more severe grade of rejection that eventually developed. Our experience was consistent with other VCAs in that donor specific antibodies did not develop, despite a severe Banff Grade. Consistent use of topical calcineurin inhibitor based immunosuppression on the allograft skin may be helpful in warding off future episodes, as our patient has been rejection free now for 18 months. To date, no histologic signs of chronic rejection were present on 2-year protocol surveillance biopsy. We have added rapamycin to the current drug regimen, with concurrent reduction of tacrolimus dosing for renal protection, which has been demonstrated in cardiac transplantation to deter the intimal hyperplasia/vasculopathy associated with chronic rejection.

Metagenomic characterization of influenza virus: bacteria super-infections associated with death and survival

Josh Klonoski, MD PhD ${ }^{1}$, Matthew Williams and Victor Huber ${ }^{1}$ The University of Utah School of Medicine

OBJECTIVES/SPECIFIC AIMS: To better understand host, viral and bacterial responses underlying disparate outcome. METHODS/ STUDY POPULATION: We utilized metagenomic analysis of 559 genes, the NanoString nCounter Immunology Panel-Plus kit and FFPE mouse lungs from a published BSI time course. RESULTS/ ANTICIPATED RESULTS: Results show an overall increased level of gene expression during BSIs associated with survival when compared to gene expression during peak viral titers. Early viral clearance and the presence of S. pyogenes in the lungs of TX98 infected lungs 24 hours after BSI was confirmed. Host responses tied to differences in early viral detection and clearance consisted of RIG-I, OAS, TLR3, TLR8 and TLR9. Key changes were noted in the expression of type I and II interferons, complement proteins, cytokines, chemokines, Fc receptors, scavenger receptors, the immunoproteasome and genes associated with T, B, Treg and NK cell activation. Interestingly, there was no significant increase in host antimicrobial peptides during BSIs associated with survival while CAMP and Nos2 were significantly increased $24 \mathrm{hrs}$ prior to death in lethal BSIs. DISCUSSION/ SIGNIFICANCE OF IMPACT: To our knowledge this is the first side by side metagenomics study of influenza BSIs associated with death and survival. Results offer mechanistic insight into clinical outcomes.

3373

\section{Modulation of Hedgehog Signaling Alters Immune Infiltration in Pancreatic Cancer}

Nina Steele ${ }^{1}$, Valerie Irizarry-Negron, Veerin Sirihorachai, Samantha Kemp, Eileen Carpenter, Christopher Halbrook, Costas Lyssiotis, Filip Bednar, Timothy Frankel, Benjamin Allen and Marina Pasca di Magliano

${ }^{1}$ University of Michigan School of Medicine

OBJECTIVES/SPECIFIC AIMS: Pancreatic ductal adenocarcinoma (PDA) has a dismal 5-year survival rate of 9\%, making this disease one of the deadliest human malignancies (https://seer.cancer.gov/). Primary barriers to the treatment of pancreatic cancer include extensive stromal interactions and sustained immune suppression. Aberrant Hedgehog $(\mathrm{HH})$ pathway activity is a hallmark of pancreatic tumorigenesis. Tumor-derived $\mathrm{HH}$ ligands signal in a paracrine fashion to the surrounding stroma to influence tumor growth. Expression of $\mathrm{HH}$ ligands increases during PDA progression, and previous work has shown that genetic deletion of Sonic HH (Shh) from the epithelium of mice with pancreatic tumors results in increased Indian $\mathrm{HH}$ (Ihh) expression. This research aims to investigate the translational impact of changes in immune infiltration following deletion of IHH in a preclinical mouse model of pancreatic cancer. METHODS/STUDY POPULATION: Ihh was deleted in tumor cells lines (IhhKO) derived from a genetically engineered mouse model of pancreatic cancer (LSL-KrasG12D/+;LSL-TrpR270H;P48-Cre), using CRISPR/Cas-9 gene editing to assess the role of Ihh in the tumor microenvironment. The level of $\mathrm{HH}$ signaling was determined using tumor cell co-cultures with GlillacZ fibroblasts (derived from mice with a lacZ reporter allele knocked into the Gli1 locus), in which Beta Galactosidase activity serves as a readout for $\mathrm{HH}$ signaling. WT and IhhKO tumor cells were orthotopically transplanted into the pancreas of syngeneic C57BL/6 mice. Human pancreas samples were obtained from surgical resection of pancreatic adenocarcinoma, or fine needle biopsy procedure (FNB). Immune profiling of mouse and human pancreatic tumors was performed using Cytometry Timeof-Flight analysis (CyTOF), and tumor composition was analyzed by single-cell RNA sequencing (scRNA seq). In vitro cultures with pancreatic fibroblasts treated with either WT or IhhKO tumor cell conditioned media (CM) were cultured with bone-marrow derived macrophages to assess tumor crosstalk. RESULTS/ANTICIPATED RESULTS: Tumor cells lacking Ihh were generated through CRISPR/ Cas- 9 deletion, and this was confirmed by qRT-PCR. Co-culture of IhhKO tumor cells with GlillacZ fibroblasts results in decreased Gli1 expression both in vitro and in vivo. Immune profiling revealed that tumors lacking Ihh have significantly fewer tumor associated macrophages $(\mathrm{CD} 11 \mathrm{~b}+/ \mathrm{F} 4 / 80+/ \mathrm{CD} 206+)$, resulting in decreased presence of immunosuppressive factors such as arginase 1 and PDL1. Immune phenotyping of human pancreatic tissues revealed similar populations of immunosuppressive myeloid cells present in tumors. In vitro co-cultures demonstrated that, in the presence of bonemarrow derived macrophages, immunosuppressive IL- 6 production was reduced in pancreatic fibroblasts cultured with IhhKO-CM, as compared to fibroblasts cultured with WT-CM, providing mechanistic insight into the in vivo phenotype observed. Further, scRNA seq analysis suggests that modulation of $\mathrm{HH}$ signaling in the tumor microenvironment alters chemokine and immunomodulatory signaling pathways driven by fibroblasts in the pancreatic tumor microenvironment. DISCUSSION/SIGNIFICANCE OF IMPACT: HH signaling in pancreatic fibroblasts contributes to the establishment of an immune suppressive environment in pancreatic cancer. Combining methods to target $\mathrm{HH}$ signaling and immune checkpoint therapy has translational potential in treating pancreatic cancer patients.

\section{4}

\section{Naltrexone as a Diagnostic Tool in Ocular Neuropathic Pain}

Nicholas Fowler Dr. ${ }^{1}$, Romulo Albuquerque ${ }^{2}$, Jooyoung Cho ${ }^{2}$, Nicholas Bell ${ }^{2}$, Paras Vora ${ }^{2}$ and Greg Botzet ${ }^{2}$

${ }^{1}$ University of Kentucky Center for Clinical and Translational Science and ${ }^{2}$ University of Kentucky College of Medicine

OBJECTIVES/SPECIFIC AIMS: The study aims to track and correlate ocular neuropathic symptoms, corneal sensitivity and dry-eye like pain, after scleral buckle and posterior vitrectomy surgeries. The goal is to identify a population of patients that receive these retinal surgeries that experience ocular neuropathic pain. METHODS/ STUDY POPULATION: Methods - Prospective and Retrospective cohort studies were designed with the follow cohorts: scleral buckle, posterior vitrectomy, and control. Typical follow up for SB/PV 